\title{
Winding uplifts and the challenges of weak and strong SUSY breaking in AdS
}

\author{
Arthur Hebecker and Sascha Leonhardt \\ Institute for Theoretical Physics, Heidelberg University, \\ Philosophenweg 19, 69120 Heidelberg, Germany \\ E-mail: a.hebecker@thphys . uni-heidelberg.de, \\ s.leonhardt@thphys.uni-heidelberg.de
}

ABSTRACT: We discuss the problem of metastable SUSY breaking in the landscape. While this is clearly crucial for the various de Sitter proposals, it is also interesting to consider the SUSY breaking challenge in the AdS context. For example, it could be that a stronger form of the non-SUSY AdS conjecture holds: it would forbid even metastable non-SUSY AdS in cases where the SUSY-breaking scale is parametrically above/below the AdS scale. At the technical level, the present paper proposes to break SUSY using the multi-cosineshaped axion potentials which arise if a long winding trajectory of a 'complex-structure axion' appears in the large-complex-structure limit of a Calabi-Yau orientifold. This has been studied in the context of 'Winding Inflation', but the potential for SUSY breaking has not been fully explored. We discuss the application to uplifting LVS vacua, point out the challenges which one faces in the KKLT context, and consider the possibility of violating the non-SUSY AdS conjecture in the type-IIA setting of DGKT.

KEYWORDS: Flux compactifications, Superstring Vacua, Supergravity Models, Supersymmetry Breaking

ArXiv EPrint: 2012.00010 


\section{Contents}

1 Introduction and summary 1

1.1 Weak and strong SUSY breaking in the landscape 1

1.2 Weak SUSY breaking from a winding uplift 3

2 The uplifting potential 5

2.1 Winding setup 5

2.2 Sub-leading terms 6

2.3 The axion potential $\quad 7$

2.4 Winding in a multi-axion field space 11

3 Uplifting AdS vacua $\quad 12$

$\begin{array}{lll}3.1 & \text { Large volume scenario } & 12\end{array}$

$\begin{array}{lll}3.2 & \text { The KKLT AdS vacuum } & 14\end{array}$

$\begin{array}{lll}3.3 & \text { DGKT-type vacua } & 16\end{array}$

$\begin{array}{lll}4 & \text { Conclusion } & 19\end{array}$

\section{Introduction and summary}

\subsection{Weak and strong SUSY breaking in the landscape}

Explicitly realizing de Sitter vacua in string theory is a long-standing challenge. The most popular approaches $[1,2]$ start with an AdS solution with stabilized moduli and promote it to a de Sitter vacuum by a so-called 'uplift'. In $4 \mathrm{~d}$ supergravity language, such effects are classified as $F$-term or $D$-term uplifts (see e.g. [3-18] and [19-25] respectively). All known models share a certain degree of complexity, which has lead to fundamental criticism [26] and the proposal of corresponding no-go theorems [27-29]. If string theory really has a problem with de Sitter, one may wonder whether the SUSY-breaking uplift is its true source.

In particular, the anti-D3-brane uplift of KKLT [1] tends to be uncomfortably high compared to the depth of the underlying AdS vacuum [30]. It has been argued that a potentially fatal 'singular-bulk problem' results [31]. ${ }^{1}$ Combining these observations with a possible general unease about SUSY-breaking uplifts, one might suspect more concretely that parametrically small SUSY breaking is problematic in string theory compactifications. In this work, we will try to construct such small uplifts using the tuning power of the complex-structure landscape.

\footnotetext{
${ }^{1}$ We do not enter the interesting debate about the 10d description of KKLT (see e.g. [30, 32-37]) since we believe that this is not going to invalidate the construction. We also note but do not discuss further the recently considered issues of throat-instabilities [38-40] and tadpole constraints [41] (see however [42]). Yet another line of attack is [43].
} 
Before describing our approach, let us briefly consider existing and suggest some further Swampland constraints relevant in this context. First, the non-SUSY AdS conjecture states that no stable non-supersymmetric (4d) AdS exists in string theory [44, 45]. This is interesting for us since a small SUSY-breaking uplift on the basis of a SUSY AdS vacuum might provide a counterexample.

A logically possible, much stronger conjecture would be one forbidding metastable nonSUSY AdS. ${ }^{2}$ One may call this 'Absolute non-SUSY AdS Conjecture'. In such a strong formulation this conjecture is in conflict with the constructions of $[46,48-51] .^{3}$ It then remains an interesting question whether some softened form of such a conjecture has a chance of being true. Most naively, one may think of flux compactifications where AdS and KK scale coincide, $R_{\mathrm{AdS}} \sim R_{\mathrm{KK}}$. If SUSY is broken by the compactification, one additionally expects $M_{\text {SUSY }} \sim R_{\mathrm{KK}}^{-1}$. It may then turn out to be difficult or impossible to escape the prediction that SUSY-breaking and AdS scale are related. ${ }^{4}$

Concretely, one might expect that metastable non-SUSY AdS with $M_{\text {SUSY }} \gg R_{\text {AdS }}^{-1}$ is forbidden. Let us call this the 'Strong-SUSY-Breaking Conjecture'. If this conjecture were false and the Swampland dS conjecture true, then a natural place for observers like us to find themselves in would be universes with a small negative cosmological constant. In other words, the 'Strong-SUSY-Breaking Conjecture' removes an anthropically interesting part of the multiverse. ${ }^{5}$

By contrast, one can also consider a conjecture stating that metastable non-SUSY AdS with $M_{\text {SUSY }} \ll R_{\text {AdS }}^{-1}$ is forbidden. This could be called the 'Weak-SUSY-Breaking Conjecture'. It is motivated by the difficulty, mentioned above, to make the anti-D3-uplift of KKLT as small as desired. We will see below that our proposal applied to the DGKT vacuum [57] may provide a counterexample to the latter ('Weak') but not to the former ('Strong') SUSY-Breaking Conjecture.

Clearly, all of the above is strongly affected if one takes the existence of the LVS AdS vacuum for a fact, even if this vacuum were only metastable. The 'Strong-SUSY-Breaking Conjecture' then immediately falls and it is likely that, through an appropriate uplift (for example the one proposed in this paper), the dS conjecture also fails. If the Winding Uplift we suggest turns out not to work, the 'Weak-SUSY-Breaking Conjecture' may coexist with LVS AdS vacua.

We also note that a conjecture against the separation of KK and AdS scale [58] (see also [33]) would, if true, change much of the discussion above. We dismiss this for the purpose of this paper, expecting the KKLT AdS vacuum with the standard fine tuning of $W_{0}[59]$ (or its specific realization in [60]) to provide a counterexample.

\footnotetext{
${ }^{2}$ By metastable we mean that the local decay rate satisfies $\Gamma \ll R_{\text {AdS }}^{-1}$. In spite of the fact that global metastable AdS decays instantaneously [46, 47], a patch of such a metastable AdS might nevertheless exist, e.g. as a cosmologically created bubble.

${ }^{3}$ The recent discussions of $[50,51]$ (based on [52]) suggest that their solutions are stable. However, it is not clear to us to which extent non-perturbative instabilities can be excluded.

${ }^{4} \mathrm{We}$ should remind the reader of the possibility of meta-stable AdS compactifications of non-SUSY string theories (the super-critical or the $O(16)^{2}$ heterotic string, see e.g. [53-55] and refs. therein). If such a compactification can be realized and if the AdS and string scale can be parametrically separated, our motivation for $M_{\text {SUSY }} \sim R_{\mathrm{KK}}^{-1}$ fails.

${ }^{5}$ While our world appears not to belong to this part, even this is not entirely certain [56].
} 


\subsection{Weak SUSY breaking from a winding uplift}

Let us now turn to the description of our technical work. We follow the original proposal by Saltman and Silverstein [3] to realize an uplift by finding metastable local minima in the complex-structure scalar potential. We will use the tuning-power of the complexstructure-based flux landscape to ensure that the corresponding $F$-term is small and the SUSY breaking is controlled. Our method of choice are the multi-cosine-shaped axion potentials, in the spirit of [61]. Specifically, several cosine terms are superimposed if a long winding trajectory of a 'complex-structure axion' appears in the large-complex-structure limit of a Calabi-Yau orientifold. This has been studied in the inflationary context as 'Winding Inflation' [62] (see also [63-66]), but the potential of this method for realizing weak SUSY breaking with long lifetimes has not been analyzed in detail. We will comment on the technically related uplifting suggestions of [67-69] in a moment.

We consider type-IIB CY orientifold compactifications with the complex structure moduli $u$ and $v$ at large-complex-structure, i.e. $\operatorname{Im} u, \operatorname{Im} v \gg 1$. In this limit, the Kähler potential does not depend on $\operatorname{Re} u$ and $\operatorname{Re} v$, such that a shift symmetry arises. It is only broken by the flux superpotential. We may choose fluxes $M$ and $N$ in such a way that only the linear combination $M u+N v$ appears in the superpotential. As a result, one linear combination of $\operatorname{Re} u$ and $\operatorname{Re} v$ is left unstabilized. We parameterize this direction in field space by $\varphi \equiv \operatorname{Re} v$.

The leading corrections to the large-complex-structure expressions for Kähler and superpotential are of the form $\exp (i u)$ and $\exp (i v)$. Both terms depend on the unstabilized axion $\varphi$ and their magnitude is governed by the stabilized values of the saxions: $\exp (i u) \propto \exp \left(-\operatorname{Im} u_{0}-i N / M \varphi\right)$ and $\exp (i v) \propto \exp \left(-\operatorname{Im} v_{0}+i \varphi\right)$. We may tune the saxion values $\operatorname{Im} u_{0}$ and $\operatorname{Im} v_{0}$ in such a way that the two terms are comparable, suppressed by an expansion parameter $\epsilon \equiv \exp \left(-\operatorname{Im} u_{0}\right) \sim \exp \left(-\operatorname{Im} v_{0}\right) \ll 1$. Their relative magnitude is then measured by a parameter $\alpha \propto \exp \left(\operatorname{Im} u_{0}-\operatorname{Im} v_{0}\right)=\mathcal{O}(1)$.

The resulting $F$-term scalar potential of the axion is, schematically, of the simple form

$$
V(\varphi)=\frac{g_{s}}{\mathcal{V}^{2}} \epsilon^{2}[\cos (\varphi)-\alpha \cos (N / M \varphi)]^{2} .
$$

Here, we introduced the string coupling $g_{s}$ and the CY volume $\mathcal{V}$ as they usually appear in an $F$-term potential. Without loss of generality, we assume $M / N<1$. In the regime $M^{2} / N^{2} \lesssim \alpha \lesssim 1$, the $F$-term potential develops non-trivial local minima, e.g. at some $\varphi=\varphi_{*}$ (cf. figure 1). The value of the potential at the minimum takes the form $V\left(\varphi_{*}\right) \sim$ $g_{s} \epsilon^{2} \gamma^{2} / \mathcal{V}^{2}$, where for $\varphi_{*}=0$ we define $\gamma \equiv 1-\alpha$. This can be tuned small if, as we will discuss in the paper, $\alpha$ is scanned sufficiently finely in the landscape such that $\gamma \ll 1$ can be realized. The height of the potential barrier separating the metastable from the global minimum is $V_{\text {wall }} \sim g_{s} \epsilon^{2} / \mathcal{V}^{2}$. This remains sizable even at very small $\gamma$. Hence, the uplifting height $\Delta V$ and the height of the barrier $V_{\text {wall }}$ can be separated parametrically.

Let us turn to possible applications of the mechanism just described in concrete settings: it is straightforward to apply it in the large volume scenario. Tuning the value of $\epsilon \gamma$ against the value of the LVS AdS cosmological constant, one may consistently uplift the vacuum to de Sitter. By contrast, uplifting a KKLT SUSY-AdS vacuum using the 


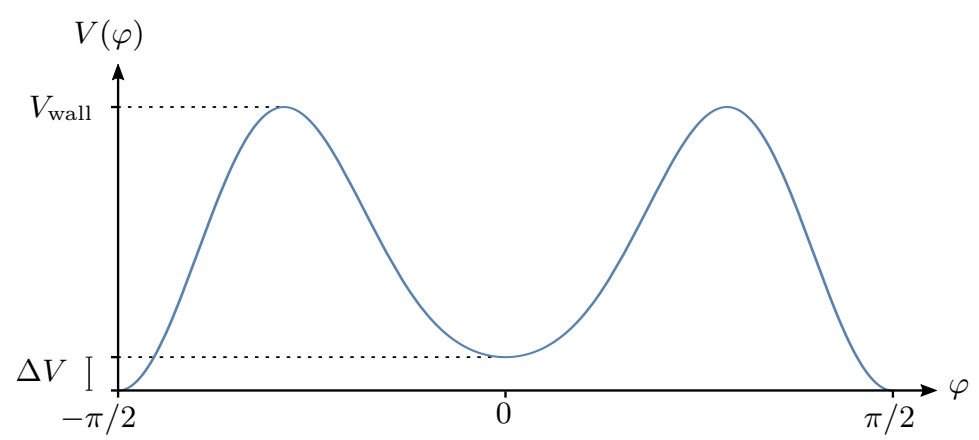

Figure 1. The axion potential (1.1) for $N / M=3$. There is a minimum at $\varphi_{*}=0$ with $\Delta V \equiv$ $V(0) \propto g_{s} \epsilon^{2} \gamma^{2} / \mathcal{V}^{2}, \gamma \ll 1$, while the potential scales as $g_{s} \epsilon^{2} / \mathcal{V}^{2}$ in general.

minimal setup just discussed is problematic. The difficulties one encounters are related to the smallness of the superpotential $W_{0}$, which is required for $10 \mathrm{~d}$ supergravity control in KKLT. This smallness spoils the stabilization of the saxions as discussed above. The situation is not hopeless if one goes to the boundary of parametric control or involves more than two axions, but we have to leave a detailed study to future work. Finally, we consider supersymmetric AdS vacua in type IIA as studied by DGKT [57]. This setting naturally gives rise to unstabilized axions in an otherwise fully supersymmetrically stabilized background. Only a single linear combination of RR axions is fixed. The superpotential resulting from non-perturbative corrections directly realizes our winding scenario with multiple axions [70]. We expect there is in general enough tuning power in this setting to find low-lying local minima protected by parametrically high barriers. While these uplifts are necessarily small, they may provide a way to turn DGKT solutions into stable non-SUSY AdS vacua.

Before closing our introduction, let us comment in more detail on earlier related work. First, we note that 'instantonic' terms have been used in various approaches to constructing de Sitter vacua, e.g., in racetrack or STU models [71-78]. In some cases, these are one-step constructions, without the separation in AdS stabilization and uplift. A distinguishing feature of our approach is the complex-structure origin of the instantonic uplifting effect. This may allow a fully explicit implementation of our scenario, along the lines of the approach of [60] to constructing a small superpotential from the interplay of instantonic terms. We also note that combining a periodic and a linear potential for a complexstructure axion was suggested as an uplifting mechanism in [67]. Moreover, the interplay of different periodic terms in axion potentials has recently been discussed in other contexts: the 'drifting monodromies' scenario in compactifications involving multi-throat systems [68] gives rise to a superpotential of the same form as we use in this work. There, the particular no-scale form of the Kähler potential prevents a direct application to uplifting. ${ }^{6}$ A closely related but slightly more speculative uplifting idea nevertheless arises in the setting of [68]. The authors of [69] give a pure IR argument on how the QCD pion potential at general

\footnotetext{
${ }^{6}$ Due to the no-scale structure only the holomorphic part of the $F$-term enters the scalar potential. This leads to the same issues of implementing our mechanism as will be discussed in section 3.2. No-scale breaking effects may resolve this problem.
} 
$\theta$-angle generates a multi-cosine-shaped scalar potential which possesses non-zero minima. They discuss how this may naturally uplift the IR theory.

Our paper is organized as follows. Section 2 presents the SUSY-breaking mechanism just discussed in detail, including a short introduction to the winding idea, a discussion of all relevant sub-leading corrections and an analysis of the axion potential induced. We describe how this may be used to uplift AdS vacua of various origins (LVS, KKLT and DGKT) in section 3. Finally, we conclude in section 4.

\section{The uplifting potential}

\subsection{Winding setup}

Let us briefly introduce the winding scenario of [62]. It is formulated in a region of moduli space where two distinguished complex structure moduli $u$ and $v$ are 'at large complex structure': $\operatorname{Im} u, \operatorname{Im} v \gg 1$. The Kähler potential is then approximately independent of $\operatorname{Re} u$ and $\operatorname{Re} v$, leading to a shift symmetry. Such approximate complex-structure shift symmetries have been studied in the inflationary context in many papers, see e.g. [79-87].

In the winding scenario, this shift symmetry is broken by the flux superpotential in such a way that a single axion-like field emerges. This field corresponds to a long, winding trajectory on the torus parameterized by $(\operatorname{Re} u, \operatorname{Re} v)$. Concretely, a flux choice is made such that the complex-structure superpotential and the full Kähler potential take the form

$$
\begin{aligned}
W_{\mathrm{cs}} & =\widetilde{W}_{0}(z)+f(z)(M u+N v)+W_{\mathrm{sub}}(z, u, v), \\
K & =K_{\mathcal{V}}-\ln (k(z, \bar{z}, \operatorname{Im} u, \operatorname{Im} v))+K_{\mathrm{sub}}(z, \bar{z}, u, \bar{u}, v, \bar{v}) .
\end{aligned}
$$

Here, $K_{\mathcal{V}}$ is the Kähler moduli Kähler potential. The variable $z$ represents the axio-dilaton together with all complex structure moduli, except for $u$ and $v$. Correspondingly, $-\ln (k)$ is the sum of axio-dilaton and complex-structure Kähler potential. ${ }^{7}$ The expressions $W_{\text {sub }}$ and $K_{\text {sub }}$ stand for terms that are sub-leading w.r.t.

$$
W_{0}(z, u, v) \equiv \widetilde{W}_{0}(z)+f(z)(M u+N v),
$$

and

$$
K_{0} \equiv K_{\mathcal{V}}-\ln (k)
$$

These sub-leading terms are suppressed by factors $\exp (i u)$ or $\exp (i v)$ and arise as corrections to the periods $\int \Omega$ of the large-complex-structure geometry. We will specify these sub-leading terms in section 2.2. Crucially, by our flux choice $u$ and $v$ appear in $W_{0}$ only in the linear combination $M u+N v$, where $M$ and $N$ are integer flux numbers. Note that we do not require large hierarchies in fluxes as will become clear later. Thereby, we avoid any potential issues arising in winding scenarios with large flux hierarchies $[70,87]$.

\footnotetext{
${ }^{7}$ To be more explicit about the axio-dilaton $\tau$, one could replace $\{z\} \rightarrow\{\tau, z\}$, such that $-\ln (k(z, \bar{z}, \operatorname{Im} u, \operatorname{Im} v)) \rightarrow-\ln (-2 \operatorname{Im} \tau)-\ln (\tilde{k}(z, \bar{z}, \operatorname{Im} u, \operatorname{Im} v))$.
} 
To analyze the leading-order $F$-terms $F_{0, i} \equiv\left(\partial_{i}+K_{0, i}\right) W_{0}$, it is convenient to change variables from $u, v$ to

$$
\psi \equiv M u+N v, \quad \phi \equiv v .
$$

The $F$-term conditions then read

$$
\begin{aligned}
F_{0, z} & =\left(\partial_{z} K_{0}\right) W_{0}+\partial_{z} \widetilde{W}_{0}+\left(\partial_{z} f\right) \psi=0, \\
F_{0, \psi} & =\left(\partial_{\psi} K_{0}\right) W_{0}+f=0, \\
F_{0, \phi} & =\left(\partial_{\phi} K_{0}\right) W_{0}=0 .
\end{aligned}
$$

While the equations for $z$ and $\psi$ are in general complex, the equation for $\phi$ is real (up to an overall phase). The SUSY conditions $F_{0, i}=0$ therefore fix

$$
z=z_{0}, \quad \psi=\psi_{0}, \quad \operatorname{Im} \phi=\operatorname{Im} \phi_{0}
$$

while $\operatorname{Re} \phi$ remains unstabilized. As a result, the imaginary parts of the original fields $u$ and $v$ are also fixed,

$$
\operatorname{Im} u=\operatorname{Im} u_{0}, \quad \operatorname{Im} v=\operatorname{Im} v_{0},
$$

while only one linear combination of their real parts is stabilized. It will prove convenient to redefine the fields $z, \psi$ and $\phi$ according to

$$
z \rightarrow z_{0}+z, \quad \psi \rightarrow \psi_{0}+\psi, \quad \phi \rightarrow \phi_{0}+\phi
$$

such that $z=\psi=\operatorname{Im} \phi=0$ in the leading-order vacuum. (We set $\operatorname{Re} \phi_{0} \equiv 0$, that is we do not shift the unstabilized field.) Since we do not apply this shift to $u$ and $v$, the relations (2.4) must be appropriately corrected:

$$
\psi \equiv M u+N v-\psi_{0}, \quad \phi \equiv v-\phi_{0} .
$$

\section{$2.2 \quad$ Sub-leading terms}

Sub-leading terms stabilize $\operatorname{Re} \phi$ and correct the vacuum values of the other fields. For the complex-structure superpotential, we have $[88]^{8}$

$$
\begin{aligned}
W_{\mathrm{cs}} & =W_{0}+W_{\mathrm{sub}}=W_{0}(z, u, v)+A(z) e^{i u}+B(z) e^{i v}+\ldots \\
& =W_{0}(z, \psi)+A(z) e^{-\operatorname{Im} u_{0}} e^{i \operatorname{Re} \psi_{0} / M} e^{i(\psi-N \phi) / M}+B(z) e^{-\operatorname{Im} v_{0}} e^{i \phi}+\ldots \\
& =W_{0}(z, \psi)+\epsilon\left[\mathcal{A}(z, \psi, \operatorname{Im} \phi) e^{-i N \varphi / M}+\mathcal{B}(z, \operatorname{Im} \phi) e^{i \varphi}\right]+\mathcal{O}\left(\epsilon^{2}\right)
\end{aligned}
$$

where

$$
\epsilon \equiv e^{-\operatorname{Im} u_{0}} \quad \text { and } \quad \varphi \equiv \operatorname{Re} \phi .
$$

\footnotetext{
${ }^{8}$ In fact, the underlying structure of corrections to the large-complex-structure expressions for periods holds both in the 3 -fold and the 4-fold case. Thus, our discussion immediately applies to the more general F-theory setting. Note that prefactors like $A(z)$ may be viewed as arising from a full resummation of terms suppressed by $\exp ($ inz $), n \in \mathbb{N}$.
} 
In (2.11), we have simply applied the field definitions from (2.9). Then, in (2.12), we have absorbed all factors depending on fields that are stabilized in leading order in the two coefficients

$$
\begin{aligned}
\mathcal{A}(z, \psi, \operatorname{Im} \phi) & =A(z) e^{i \operatorname{Re} \psi_{0} / M} e^{i \psi / M+N \operatorname{Im} \phi / M}, \\
\mathcal{B}(z, \operatorname{Im} \phi) & =B(z) e^{\operatorname{Im} u_{0}-\operatorname{Im} v_{0}} e^{-\operatorname{Im} \phi} .
\end{aligned}
$$

As a result, our expression for $W_{\text {sub }}$ in (2.12) is manifestly a sum of two exponentials with different periodicities in the light axionic variable $\varphi$. While this is now somewhat hidden, $W_{\text {sub }}$ of course remains holomorphic in $\phi$.

The large complex structure regime implies $\epsilon \ll 1$. Furthermore, we assume that by landscape-tuning

$$
\operatorname{Im} u_{0} \simeq \operatorname{Im} v_{0} \quad \text { or } \quad|\mathcal{B}(0) / \mathcal{A}(0)| \propto e^{\operatorname{Im} u_{0}-\operatorname{Im} v_{0}}=\mathcal{O}(1),
$$

such that the two sub-leading terms in (2.12) are comparable. We will specify the required $\mathcal{O}(1)$-ratio more precisely below.

We also add the relevant corrections to the Kähler potential:

$$
\begin{gathered}
K=K_{0}+K_{\text {sub }}=K_{0}(z, \bar{z}, \operatorname{Im} u, \operatorname{Im} v)+K_{\text {sub }}(z, \bar{z}, \phi, \bar{\phi}, \psi, \bar{\psi}) \\
K_{\text {sub }}=\left(\widetilde{A}(z, \bar{z}, \operatorname{Im} u, \operatorname{Im} v) e^{i u}+\widetilde{B}(z, \bar{z}, \operatorname{Im} u, \operatorname{Im} v) e^{i v}+\text { c.c. }\right)+\ldots \\
=\epsilon\left[\widetilde{\mathcal{A}}(z, \bar{z}, \operatorname{Re} \psi, \operatorname{Im} \psi, \operatorname{Im} \phi) e^{-i N \varphi / M}\right. \\
\left.\quad+\widetilde{\mathcal{B}}(z, \bar{z}, \operatorname{Im} \psi, \operatorname{Im} \phi) e^{i \varphi}+\text { c.c. }\right]+\mathcal{O}\left(\epsilon^{2}\right) .
\end{gathered}
$$

Here $\widetilde{\mathcal{A}}$ and $\widetilde{\mathcal{B}}$ are defined similarly to $\mathcal{A}$ and $\mathcal{B}$. Note that our treatment of $K_{\text {sub }}$ as a sub-leading correction relies on the fact that the prefactors $\widetilde{A}$ and $\widetilde{B}$ depend on $\operatorname{Im} u$ and $\operatorname{Im} v$ only polynomially [88].

\subsection{The axion potential}

We now turn to the scalar potential induced by the sub-leading terms. Our analysis of the back-reaction on the leading-order solution simplifies the discussion presented in [62]. This will be useful for the generalization to small $W_{0}$ required later on.

It will be convenient for the moment to include $\phi$ and $\psi$ in the set of complex structure moduli denoted by $z^{i}, i=1, \ldots, n$. So the index ' $i$ ' now runs over all complex structure moduli and the axio-dilaton. We also shift all fields such that the leading-order vacuum is at $z^{i}=0$ for all $i$.

Using the no-scale structure of the Kähler sector, the scalar potential takes the form ${ }^{9}$

$$
V=e^{K} K^{i \bar{\jmath}} F_{i} \bar{F}_{\bar{\jmath}}
$$

\footnotetext{
${ }^{9}$ To be precise, for this simple expression to be correct the 2 -form-axion superfields (associated with non-zero $h_{-}^{1,1}$ ) have to be set to zero. In general, these fields appear in the Kähler moduli Kähler potential in combination with the axio-dilaton, which is one of our $z^{i}$. For non-zero 2-form axions, this affects the relevant $F_{i}$. Eventually, the potential is nevertheless independent of these 2-form axions because of their shift symmetry and an associated special no-scale cancellation [89, 90].
} 
where at zeroth order in $\epsilon$ we have $F_{0, i}=0$ at $z^{i}=0$ (2.5). At linear order in $\epsilon, F_{i}$ receives a correction $\delta F_{i}$ coming both from corrections to $K$ and $W$ :

$$
\delta F_{i}=\partial_{i} K_{0} W_{\mathrm{sub}}+\partial_{i} K_{\mathrm{sub}} W_{0}+\partial_{i} W_{\mathrm{sub}} .
$$

But it would be too naive to simply replace $F_{i}$ in $(2.17)$ by $\delta F_{i}$. The reason is that the $z^{i}$ back-react. This back-reaction is small, $z^{i} \sim \epsilon$, since $\delta F_{i} \sim \epsilon$. We may thus Taylor expand in $z^{i}$ and evaluate (2.17) with the replacement

$$
F_{i} \quad \longrightarrow \quad F_{i j} z^{j}+F_{i \bar{\jmath}} \bar{z}^{\bar{j}}+\delta F_{i} .
$$

Here, $F_{i j}=\partial F_{0, i} / \partial z^{j}$ and similarly for $F_{i \bar{\jmath}}$. Since we are only interested in calculating $V$ at the order $\epsilon^{2}$, the $z^{i}$ dependence of $\exp (K)$ and $K^{i \bar{\jmath}}$ in (2.17) may be disregarded. Moreover, the dependence of the small quantities $\delta F_{i}$ on the small parameters $z^{i}$ is irrelevant since it gives sub-leading terms in the $\epsilon$-expansion.

In fact, the last statement comes with a crucial exception: namely, our light axion $\varphi$ is now simply the real part of one of the $z^{i}$, and this field is not stabilized at leading order. Hence, in contrast to what was assumed about the generic $z^{i}$ above, this particular field excursions can take $\mathcal{O}(1)$ values. However, $\varphi$ appears in $\delta F_{i}$ and only there. Thus, our final result is (2.17) with the replacement (2.19) and the extra prescription that $\delta F_{i}$ should be evaluated in full precision w.r.t. $\varphi$ while keeping all other fields at their leading-order vacuum value, $z^{i}=0$.

To proceed, let us view (2.17) as the length squared of the complex vector $F_{i}$. At the expense of doubling the index range and appropriately redefining the metric, we may view this as the length squared of a real vector:

$$
V=G^{a b} f_{a} f_{b} \quad \text { with } \quad f_{a}=k_{a b} x^{b}+\delta f_{a}\left(x^{1}\right) \quad \text { and } \quad z^{i}=x^{2 i-1}+i x^{2 i} .
$$

Here, we set $x^{1} \equiv \varphi$ such that the vector $k_{a 1}$ vanishes by leading-order shift symmetry. The index range is $a, b=1, \ldots, 2 n$. The quantities $G^{a b}, f_{a}, \delta f_{a}$ and $k_{a b}$ follow from (2.17) and (2.19) by a simple rewriting in real and imaginary components.

Our potential as a function of $x^{1}$ follows from (2.20) by integrating out $x^{2}, \ldots, x^{2 n}$, which is straightforward: the first term in $f_{a}$ generically takes values in a $(2 n-1)$ dimensional subspace of the $\mathbb{R}^{2 n}$ in which $f_{a}$ and $\delta f_{a}$ live. This is a result of $k_{a 1}$ vanishing. Let us call the unit vector orthogonal to that 'allowed' subspace $\hat{e}_{a}$. In this we use the inner product defined by $G^{a b}$. The vector $f_{a}$ can now be decomposed as the sum of its projection on $\hat{e}_{a}$ and its orthogonal projection. The potential is the sum of the squares of these two vectors:

$$
V=\left|P_{\hat{e}}(f)\right|^{2}+\left|P_{\perp \hat{e}}(f)\right|^{2} .
$$

When minimizing in $x^{2}, \ldots, x^{2 n}$ at fixed $x^{1}$ the vector $k_{a b} x^{b}$ will take the value $-\left(P_{\perp \hat{e}}(\delta f)\right)_{a}$ such that the second term in (2.21) vanishes. By contrast, the $\hat{e}_{a}$-subspace is not accessible to $k_{a b} x^{b}$, so one is simply left with the square of the projection of $\delta f_{a}$ on that subspace:

$$
V=\left|P_{\hat{e}}(f)\right|^{2}=\left|P_{\hat{e}}(\delta f)\right|^{2}=\left(\hat{e}^{a} \delta f_{a}\left(x^{1}\right)\right)^{2} .
$$


The elements of $\hat{e}^{a}=G^{a b} \hat{e}_{b}$ may be calculated in terms of the vacuum values of $K_{0}$ and $W_{0}$. Given the form of $\delta F_{i}$ in (2.18), we see that the expression for the potential only contains sine and cosine terms in $\varphi$ with periodicity $2 \pi$ and $2 \pi M / N$ as inherited from the complex exponentials in $W_{\text {sub }}$ and $K_{\text {sub }}$. We may hence choose to parameterize the potential as

$$
V(\varphi)=e^{K_{0}} \kappa \epsilon^{2}\left[\cos \left(\varphi+\delta_{1}\right)-\alpha \cos \left(N \varphi / M+\delta_{2}\right)\right]^{2} .
$$

Here $\kappa$ and $\alpha$ are generically $\mathcal{O}(1)$ coefficients and $\delta_{1,2}$ are phases arising in the transition from the complex to the real parameterization. ${ }^{10}$ It will be crucial that all the above coefficients and in particular $\alpha$ are tunable if a dense discretuum of vacua on the space parameterized by the $z^{i}$ exists. For example, $\alpha$ can be tuned using the ratio of exponentials of $u_{0}$ and $v_{0}$ in (2.15).

Finally, we express the Kähler potential $K_{0}$ through string coupling $g_{s}$ and CY volume $\mathcal{V}$. Absorbing numerical as well as $\tau$-independent terms in $k(0)$ in the prefactor $\kappa$, we arrive at

$$
V(\varphi)=\frac{g_{s}}{\mathcal{V}^{2}} \kappa \epsilon^{2}\left[\cos \left(\varphi+\delta_{1}\right)-\alpha \cos \left(\frac{N}{M} \varphi+\delta_{2}\right)\right]^{2} .
$$

Without loss of generality we assume a flux ratio $N / M>1$.

To see that this potential possesses non-zero, local minima for tuned values of $\alpha$ and $\delta_{i}$, we consider the tuning $\delta_{1}=\delta_{2}=0$. One easily finds that the potential has an extremum at $\varphi=0$ with

$$
V(0)=\frac{g_{s}}{\mathcal{V}^{2}} \kappa \epsilon^{2} \gamma^{2}, \quad V^{\prime \prime}(0)=2 \frac{g_{s}}{\mathcal{V}^{2}} \kappa \epsilon^{2} \gamma\left[\frac{N^{2}}{M^{2}} \alpha-1\right] .
$$

Here we have defined $\gamma \equiv(1-\alpha)$. The extremum is a minimum for $1>\alpha>M^{2} / N^{2}$ and we may tune its potential value to be small by choosing $\gamma \ll 1$, see figure 2 . While also the second derivative becomes small, it goes to zero much more slowly: only linearly in $\gamma$.

The decay constant and mass of $\varphi$ are given by

$$
\begin{aligned}
f_{\varphi}^{2} & =K_{\phi \bar{\phi}}=\mathcal{O}(1), \\
m_{\varphi}^{2} & =V^{\prime \prime}(0) / f_{\varphi}^{2}=\mathcal{O}(1) \frac{g_{s}}{\mathcal{V}^{2}} \epsilon^{2} \gamma\left[\frac{N^{2}}{M^{2}} \alpha-1\right] .
\end{aligned}
$$

Here we disregard the fact that, strictly speaking, $K_{\phi \bar{\phi}}$ is parametrically small by being suppressed by some power of $\operatorname{Im} u_{0}=\ln (1 / \epsilon)$. Such minor effects are not essential in our context. Note that small flux ratios, $N / M-1 \ll 1$, suppress the mass of $\varphi$. For $\gamma \ll N^{2} / M^{2}-1$ the potential well around the minimum remains deep however.

It is clear that an imperfect tuning, $\delta_{i} \simeq 0$, will not endanger our ability to adjust $\alpha$ and still realize a positive minimum at parametrically small potential value. There will

\footnotetext{
${ }^{10}$ To be precise, the perturbations $\delta F_{i}(\varphi)$ in complex notation contain periodic terms $\propto e^{-i N \varphi / M}$ with coefficients $\partial_{z^{i}} K_{0} \mathcal{A}, i N \mathcal{A} / M, \partial_{z^{i}} \mathcal{A}, W_{0} i N \widetilde{\mathcal{A}} / M$ and $W_{0} \partial_{z^{i}} \widetilde{\mathcal{A}}$ as well as periodic terms $\propto e^{i \varphi}$ with coefficients $\partial_{z^{i}} K_{0} \mathcal{B}, i \mathcal{B}, \partial_{z^{i}} \mathcal{B}, W_{0} i \widetilde{\mathcal{B}}$ and $W_{0} \partial_{z^{i}} \widetilde{\mathcal{B}}$. These coefficients are generically $\mathcal{O}(1)$ when we are in the regime specified by (2.15). Due to the projection on $\hat{e}$, the final result also depends on further (second) derivative terms of the leading-order Kähler and superpotential evaluated in $z^{i}=0$.
} 

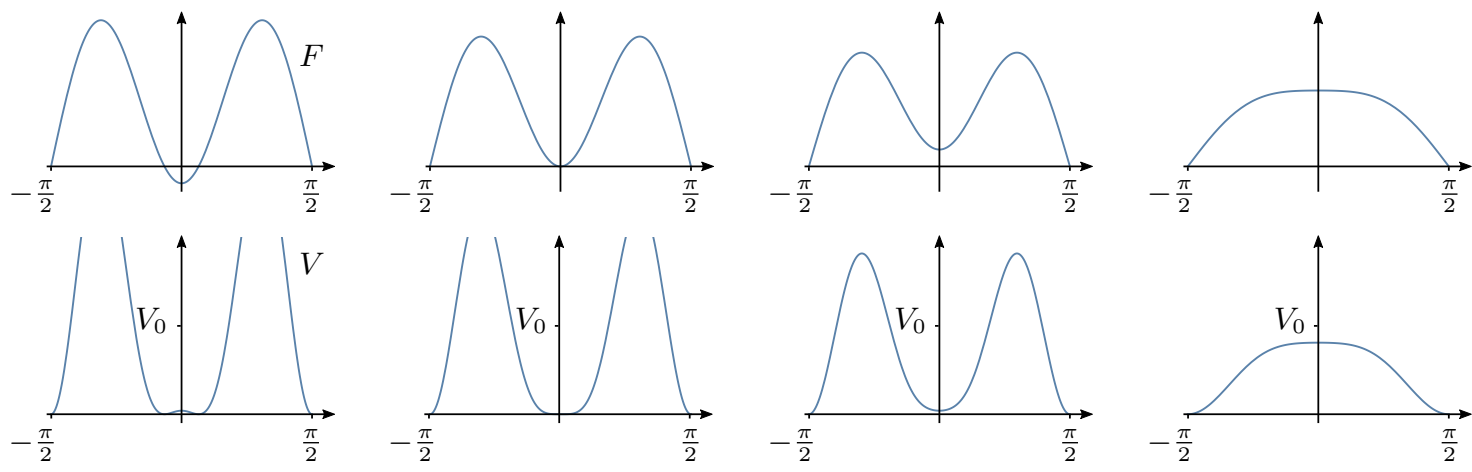

Figure 2. The $F$-term $\propto[\cos (\varphi)-\alpha \cos (3 \varphi)]$ (upper panels) and corresponding scalar potential $V_{0}[\cos (\varphi)-\alpha \cos (3 \varphi)]^{2}$ (lower panels) for $\alpha=1.2,1,0.8,0.1$ from left to right. By tuning $\alpha$ we find local minima at arbitrarily small positive value (third column). If $\alpha$ becomes too small the minima disappear (fourth column).

also be qualitatively distinct, deep minima for different values of $\delta_{i}$. One could pursue their analytic study, but this does not appear necessary at present. For what follows, we will shift the field $\varphi$ such that the non-zero minimum remains at $\varphi=0$.

With this we have arrived at one of our main technical results: we have provided an $F$-term uplift which, given enough tuning power in the complex structure landscape, can be extremely small and, in particular, small relative to the barrier protecting it from decay to supersymmetric minima.

Before closing this subsection and turning to generalizations and applications, let us summarize what our tuning requirements are precisely: first, we need a flux choice realizing the winding scenario, cf. the second term on the r.h. side of (2.2). For such a flux choice to exist, certain conditions have to be met by the integers which define the periods in the large complex structure limit (cf. eq. (6.6) of [88]). For example, terms of the type $z^{i} u$ and $z^{i} v$ have to be present. The relevant integers are mostly the triple-intersection numbers of the dual 3-fold. Second, the Kähler and superpotential have to be such that $\operatorname{Im} u$ and $\operatorname{Im} v$ are stabilized at an appropriately large value (at large complex structure). One might be concerned that this is a strong constraint since one linear combination of these moduli does not appear in $W$, where most of the tuning power resides. We note however that $K=K(u-\bar{u}, v-\bar{v}, z)$, if viewed as a function of $\operatorname{Im} u$ and $\operatorname{Im} v$, depends in detail on the values at which all the variables $z^{i}$ are stabilized. Hence the function $K(u-\bar{u}, v-\bar{v})$ can be tuned through tuning the vacuum values of the $z^{i}$. We then expect to have the full tuning power of the complex-structure landscape at our disposal. Third, we need to tune the uplift potential, specifically the constants $\alpha$ and $\delta_{1,2}$ in (2.24). Explicitly writing these constants in terms of $K, W$ and their derivatives would be cumbersome, but we have provided enough details above such that, in principle, such expressions can be derived for any given model. As explained previously for the tuning of $\operatorname{Im} u$ and $\operatorname{Im} v$, everything will depend on whether the flux landscape, viewed as a discrete set of points in the $z^{i}$-space, is dense enough. The seminal analysis of [59] and subsequent work appear to support this. Moreover, the very recent explicit analysis of [91] provides promising results concerning specifically the setup discussed in the present paper. 


\subsection{Winding in a multi-axion field space}

We may generalize to situations where multiple complex structure moduli $u^{i}(i=0, \ldots, m)$ are in the large-complex-structure limit and appear in $W$ only linearly at leading order. A possible superpotential that can arise in this case is

$$
W_{0}\left(z, u^{i}\right)=\widetilde{W}_{0}(z)+f(z)\left(\sum_{i=0}^{m} N_{i} u^{i}\right) .
$$

This is in fact similar to what happens generically in the type-IIA case to be discussed below.

Now, defining

$$
\psi \equiv \sum_{i=0}^{m} N_{i} u^{i} \quad \text { and } \quad \phi^{i} \equiv u^{i} \quad \text { for } \quad i=1, \ldots, m
$$

we find the leading-order $F$-term conditions

$$
F_{0, \psi}=\left(\partial_{\psi} K_{0}\right) W_{0}+f(z)=0, \quad F_{0, \phi^{i}}=\left(\partial_{\phi^{i}} K_{0}\right) W_{0}=0 .
$$

By the same reasoning as in section 2.1 all imaginary parts are stabilized, $\operatorname{Im} u^{i}=\operatorname{Im} u_{0}^{i}$. By contrast, only one of the real parts is fixed, $\operatorname{Re} \psi=\operatorname{Re} \psi_{0}$. The remaining $m$ axions are massless at leading order. Shifting the fields $\psi$ and $\phi^{i}$ as in (2.8), the corrected superpotential takes the form

$$
\begin{aligned}
W_{\mathrm{cs}} & =W_{0}+A_{0} e^{-\operatorname{Im} u_{0}^{0}} e^{i \frac{\operatorname{Re} \psi_{0}}{N_{0}}} e^{-i \sum_{i=1}^{m}\left(\frac{N_{i}}{N_{0}} \phi^{i}\right)}+\sum_{i=1}^{m} A_{i} e^{-\operatorname{Im} u_{0}^{i}} e^{i \phi^{i}}+\ldots \\
& \equiv W_{0}+\epsilon\left[\mathcal{A}_{0} e^{-i \sum_{i=1}^{m}\left(\frac{N_{i}}{N_{0}} \varphi^{i}\right)}+\sum_{i=1}^{m} \mathcal{A}_{i} e^{i \varphi^{i}}\right]+\mathcal{O}\left(\epsilon^{2}\right),
\end{aligned}
$$

with the $\mathcal{A}_{i}$ defined as in section 2.2. The Kähler potential is of a similar form. As long as all coefficients $e^{-\operatorname{Im} u_{0}^{i}}(i=0, \ldots, m)$ are of the same order $\epsilon$, the resulting $F$-terms may be tuned to behave analogously to figure 2 . Of course, this now occurs over a higherdimensional field space.

Another, maybe more interesting generalization arises if the superpotential of (2.27) involves different $z$-dependent prefactors [87]. We illustrate this using the 3 -axion case by assuming a superpotential of the form

$$
W_{0}\left(z, u^{i}\right)=\widetilde{W}_{0}(z)+f(z)\left(\sum_{i=1}^{3} M_{i} u^{i}\right)+g(z)\left(\sum_{i=1}^{3} N_{i} u^{i}\right) .
$$

A natural parameterization is now provided by

$$
\psi^{1} \equiv \sum_{i=1}^{3} M_{i} u^{i}, \quad \psi^{2} \equiv \sum_{i=1}^{3} N_{i} u^{i}, \quad \phi \equiv u^{3} .
$$

Crucially, only a single axion, $\varphi \equiv \operatorname{Re} \phi$, remains unstabilized [87]. Repeating the exercise of adding sub-leading corrections results in a superpotential

$$
W_{\mathrm{cs}}=W_{0}+\epsilon\left[\mathcal{A}_{1} e^{-i \frac{M_{3} N_{2}-M_{2} N_{3}}{M_{1} N_{2}-M_{2} N_{1}} \varphi}+\mathcal{A}_{2} e^{-i \frac{M_{3} N_{1}-M_{1} N_{3}}{M_{2} N_{1}-M_{1} N_{2}} \varphi}+\mathcal{A}_{3} e^{i \varphi}\right]+\mathcal{O}\left(\epsilon^{2}\right) .
$$


This may be generalized further to $m+1$ fields $u^{i}$ appearing in $m$ linear combinations in the leading-order superpotential. After integrating out heavy fields, the resulting $F$-term potential will be a periodic function of a single axion, but with many tunable parameters $\mathcal{A}_{i}$. We will discuss the possible importance of this extra tuning freedom in the application to KKLT below.

Finally, we may combine the previous generalizations by considering $m+1$ axions appearing in the superpotential in $n$ linear combinations (with $1 \leq n \leq m$ ). The result will be a sub-leading potential for $m-n+1$ axions.

\section{Uplifting AdS vacua}

In this section, we finally turn to our main goal: the uplifting of known AdS vacua to higherlying AdS and dS solutions. Our interest is in testing conjectures against non-SUSY AdS and dS models [28, 29, 44, 45].

Before turning to explicit scenarios, we want to highlight the key feature of our complex-structure $F$-term scalar potential (2.24): let $\Delta V=V_{f}-V_{t} \propto g_{s} \epsilon^{2} \gamma^{2} / \mathcal{V}^{2}$ be the difference between the non-zero false-vacuum value $V_{f}$ in the minimum $(2.25)$ and the true, global minimum at $V_{t}=0$. This $\Delta V$ is tunable via the complex structure, which we characterize by the potentially very small parameter $\gamma$. When including non-trivial effects of Kähler moduli, both $V_{f}$ and $V_{t}$ will change. Nevertheless, we expect that $\Delta V$ will remain small if $\gamma$ was tuned to a tiny value. By contrast, the potential barrier between $V_{f}$ and $V_{t}$ as well as the field distance $\Delta \varphi$ between vacua is independent of $\gamma$. This allows for a high degree of stability against false vacuum decay via Coleman-de Luccia bubble nucleation [92]. ${ }^{11}$

We now turn to explicit scenarios of uplifting.

\subsection{Large volume scenario}

The axion potential (2.24) was derived assuming $\left|W_{0}\right|=\mathcal{O}(1)$. This is consistent with the large volume scenario for Kähler moduli stabilization [2]. Thus, we may straightforwardly apply our method. Of course, many studies dedicated to uplifting the LVS exist [13, 14, 24, 25, 73, 77, 95-97]. Moreover, the warped anti-D3-brane uplift of KKLT [1] is also applicable to the LVS. However, our approach stands out because it is truly minimal: it only uses ingredients which are already present in the LVS AdS vacuum, employing the tuning power of the flux-landscape rather then extra features like matter sectors or throats.

We want to highlight [97] which is based on [98, 99]. There, a general uplift mechanism in the continuous flux approximation is presented: in the LVS fluxes are chosen such that the complex-structure $F$-terms do no longer vanish, $F_{i}=\epsilon W_{0} f_{i} \neq 0$. Here, $f_{i}$ is a unitvector in the complex-structure moduli space and $\epsilon \ll 1$ may be tuned such that the corresponding scalar potential $V=g_{s} \epsilon^{2}\left|W_{0}\right|^{2} / \mathcal{V}^{2}$ uplifts the LVS AdS vacuum. It is shown that the assumed structure of $F_{i}$ necessarily implies that one real direction of the

\footnotetext{
${ }^{11}$ We have not investigated the possibility that decays to bubbles of nothing [93] affect our non-SUSY vacuum [94]. While we do not see how this would happen in our setting, a more careful study may be warranted.
} 
complex-structure moduli space remains light. While the idea of uplifting via small SUSYbreaking $F$-terms at the cost of an additional light field is as in our mechanism, we do not require the assumption of continuous fluxes to achieve the required tuning.

The LVS requires (at least) two Kähler moduli $T_{b}$ and $T_{s}$. Since the stabilization will eventually realize a hierarchy of the corresponding four-cycle volumes, $\tau_{b} \equiv \operatorname{Re} T_{b} \gg$ $\operatorname{Re} T_{s} \equiv \tau_{s}$, only the dominant non-perturbative correction $W_{\mathrm{np}} \propto e^{-a_{s} T_{s}}$ needs to be considered. In addition, the leading perturbative $\alpha^{\prime}$-effect [100] is essential. It corrects the Kähler potential according to $-2 \ln (\mathcal{V}) \rightarrow-2 \ln (\mathcal{V}+\xi)$. Here, $\mathcal{V}=\tau_{b}^{3 / 2}-c \tau_{s}^{3 / 2}$ and, for our purposes, $\xi \sim g_{s}^{-3 / 2}$ is a constant that can be tuned by choosing the stabilized value of $g_{s}$. The resulting $F$-term scalar potential has a minimum in $\tau_{s}$, which we hence integrate out. One is then left with a potential for $\mathcal{V} \approx \tau_{b}^{3 / 2}$, which we parameterize as

$$
V_{\mathrm{LVS}}(\mathcal{V})=A \frac{g_{s} \xi\left|W_{0}\right|^{2}}{\mathcal{V}^{3}}-B \frac{g_{s}\left|W_{0}\right|^{2}}{\mathcal{V}^{3}} \ln (\mathcal{V})^{3 / 2}+\mathcal{O}\left(\frac{g_{s} \ln (\mathcal{V})^{1 / 2}}{\mathcal{V}^{3}}\right)
$$

where $A$ and $B$ are positive $\mathcal{O}(1)$-coefficients. This potential has an AdS minimum at

$$
V_{\mathrm{AdS}}=-\mathcal{O}(1) \frac{g_{s}\left|W_{0}\right|^{2} \ln (\mathcal{V})^{1 / 2}}{\mathcal{V}^{3}}
$$

with $\mathcal{V} \sim \exp \left(a_{s} \tau_{s}\right)$ and $\tau_{s} \sim \xi^{2 / 3}$. There is a mass hierarchy $m_{\tau_{b}} \ll m_{\tau_{s}}$, justifying a posteriori the procedure just described. To be precise, from (3.1) and the Kähler potential we find

$$
m_{\tau_{b}}=\mathcal{O}(1) \frac{g_{s}\left|W_{0}\right|^{2} \ln (\mathcal{V})^{1 / 2}}{\mathcal{V}^{3}}
$$

The above assumed a vanishing complex-structure $F$-term scalar potential. Our main point is in relaxing this assumption and adding the complex-structure uplift of section 2.3:

$$
V(\mathcal{V}, \varphi)=V_{\mathrm{LVS}}(\mathcal{V})+\frac{g_{s} \kappa \epsilon^{2}}{\mathcal{V}^{2}} f(\varphi)
$$

Here $f(\varphi)$ is a non-negative periodic function (cf. (2.24)) with supersymmetric minima at $f=0$ and a SUSY-breaking minimum at $f(0)=\gamma^{2}$. We choose to tune

$$
\kappa \epsilon^{2} \gamma^{2}=\mathcal{O}(1) \frac{\left|W_{0}\right|^{2} \ln (\mathcal{V})^{1 / 2}}{\mathcal{V}}
$$

where $\mathcal{V}$ is the LVS value of the volume. This tuning corresponds to an uplift to a Minkowski, shallow AdS or low-lying dS vacuum. One easily checks that such an uplift does not destabilize $\mathcal{V}$ and that a mass hierarchy $m_{\tau_{s}} \gg m_{\tau_{b}}, m_{\varphi}$ is obeyed. We hence do not need to reconsider the step of integrating out $\tau_{s}$.

The potential (3.4) describes a 2-field model with a mass ratio $m_{\tau_{b}} / m_{\varphi} \sim \sqrt{\gamma}$ (cf. (2.26) and (3.3)). The condition (3.5) may be realized with either $\gamma \ll 1$ or $\gamma=\mathcal{O}(1)$. In the first case, the complex-structure axion is lighter than the volume modulus, in the second case they have similar masses.

Note that, while the possibility of an extremely small uplift due to a tuning $\gamma \ll 1$ is a distinguishing feature of our approach, this is not required for the LVS uplift. Indeed, 
in the present case, a value $\gamma=\mathcal{O}(1)$ does not spoil the longevity of the vacuum: while the potential barrier between true AdS and false $\mathrm{dS}$ vacuum is not parametrically high compared to $\Delta V$, the distance $\Delta \varphi$ between vacua remains sufficiently large. In summary, if the tuning we assumed can be explicitly realized, our mechanism could challenge the dS conjecture [28, 29].

The analysis of this section is built on the very reasonable expectation that the SUSYbreaking AdS vacuum of the LVS approach is long-lived. It is in fact even possible that this vacuum is stable and therefore in conflict with the non-SUSY AdS conjecture [101, 102]. In either case, i.e. both for a long-lived and for a stable LVS AdS vacuum, the possibility of our small uplift to a higher-lying AdS does not improve its usefulness as a counterexample to the non-SUSY AdS conjecture. Therefore, we do not pursue this further.

Before closing the present section, we should comment on a geometric consistency issue related to the large-complex-structure limit. In this limit, the CY can be thought of as a $T^{3}$-fibration over an $S^{3}$ with the fiber volume becoming singular [103-105]. Assigning a typical radius $R$ to the torus-fiber and a radius $L$ to the base three-sphere, the imaginary parts of the complex-structure moduli (in the conventions of this paper) scale as $L / R$ in the limit $L / R \rightarrow \infty$ (cf. [80]). Since CY 2-cycles scale as $L \cdot R$ and since $R>1$ in string units is required for supergravity control, a lower bound on the volume is obtained, $\mathcal{V} \gtrsim L^{3}$. To be precise, the moduli we assumed to be at large complex structure are $u$ and $v$. We then have $\operatorname{Im} u \sim \operatorname{Im} v \sim L / R$ and find the condition

$$
\operatorname{Re} T_{b} \sim \mathcal{V}^{2 / 3} \gtrsim(\operatorname{Im} u)^{2} \sim(\operatorname{Im} v)^{2}
$$

This is easily consistent with the tuning requirement (3.5). Indeed, since $\epsilon=\exp (-\operatorname{Im} u)$, our volume is exponentially large in $\operatorname{Im} u$. It becomes even larger if $\gamma \ll 1$ or $\left|W_{0}\right| \gg 1$. For the small 4-cycle, a condition analogous to (3.6) is not obeyed so easily since Re $T_{s}$ grows only like $\ln \mathcal{V}$. It may still hold if $\gamma$ is tuned sufficiently small. But, most importantly, we expect that we do not even need to implement a geometric consistency condition like $\operatorname{Re} T_{s} \gg(L / R)^{2}$ because our large-complex-structure limit is only partial: all the complex structure moduli $z^{i}$ apart from $u$ and $v$ are not required to be at large complex structure. Hence, we may focus on geometries where the blow-up cycle governed by $T_{s}$ is geometrically separated from the specific shrinking 3 -cycles related to $u$ and $v$. In such geometries, we expect that supergravity control is straightforwardly compatible with the partial largecomplex-structure limit we require.

\subsection{The KKLT AdS vacuum}

So far we considered non-tuned flux-superpotentials: $\left|W_{\mathrm{cs}}\right| \simeq\left|W_{0}\right| \gtrsim \mathcal{O}(1)$. Let us now turn to uplifting the supersymmetric type-IIB KKLT vacuum [1] which relies on the tuning $\left|W_{\text {cs }}\right| \ll 1$. Note that our convention differs from [1] in that we denote the complexstructure superpotential by $W_{\mathrm{cs}}$ and reserve the symbol $W_{0}$ for the contribution that is leading at large complex structure: $W_{\mathrm{cs}} \equiv W_{0}+W_{\text {sub }}$. After including the Kähler modulus 
superpotential ${ }^{12}$

$$
W_{\mathrm{np}}(T)=A_{K} e^{-a T},
$$

the modulus $T$ is stabilized at a value where $e^{-a \operatorname{Re} T} \sim\left|W_{\mathrm{cS}}\right|$.

We notice that there is a problem in implementing the simple mechanism as described in section 2.3: if we consider a CY at the large-complex-structure point, we are subject to (3.6). For the realization of the KKLT scenario in type IIB, this implies

$$
\left|W_{\text {cs }}\right|=\left|W_{0}+W_{\text {sub }}\right| \sim e^{-a \operatorname{Re} T} \ll e^{-\operatorname{Im} u} \sim \epsilon .
$$

Therefore, the perturbative analysis of section 2 relying on $\epsilon \ll\left|W_{0}\right|$ breaks down.

There are two possibilities to proceed: first, one may try to find a flux choice such that $\left|W_{0}+W_{\text {sub }}\right| \ll \epsilon$. Since generically $W_{\text {sub }} \sim \epsilon$, this requires a $W_{0}$ of the same magnitude. This means that our calculational approach, which treats $W_{\text {sub }}$ as a small correction, is at the boundary of control and becomes unreliable. Nevertheless, one may hope that there are concrete models in which the qualitative features of our uplifting method survive.

Second, one may try to implement a hierarchy $\left|W_{\text {sub }}\right| \ll\left|W_{0}\right| \ll \epsilon$. This requires a fine-tuned cancellation between $\mathcal{O}(\epsilon)$-terms within $W_{\text {sub }}$. The advantage is that one can hope to maintain the method of treating $W_{\text {sub }}$ as a small correction to $W_{0}$.

In either case, the whole superpotential $W=W_{\mathrm{cs}}(\phi)+W_{\mathrm{np}}(T)$ is small, which implies that not just the real component but the whole superfield $\phi$ remains light. We expect a consistent supergravity description to exist for the light moduli $\phi$ and $T$ with

$$
W=W_{0}+W_{\mathrm{sub}}(\phi)+W_{\mathrm{np}}(T), \quad K=-3 \ln (\operatorname{Re} T)-\ln (k(\operatorname{Im} \phi))
$$

and

$$
V=e^{K}\left[K^{T \bar{T}}\left|D_{T} W\right|^{2}+K^{\phi \bar{\phi}}\left|D_{\phi} W\right|^{2}-3|W|^{2}\right] .
$$

The lightness of $\operatorname{Im} \phi$ follows from the fact that it enters $V$ only via the small superpotential $W_{\text {sub }}$ or through the prefactors $K_{\phi}, K^{\phi \bar{\phi}}$ and $e^{K}$, which multiply small quantities. Note also that corrections to $K$ are not relevant for sufficiently small $W$.

We proceed on the basis of the supersymmetric minimum at $D_{T} W=0$ and $D_{\phi} W=0$. Given our assumptions, this minimum is characterized by [1]

$$
\left|W_{\mathrm{cS}}\right| \sim\left|A_{K}\right| e^{-a \operatorname{Re} T}, \quad V_{\mathrm{AdS}} \sim-\left|W_{\mathrm{cS}}\right|^{2} .
$$

Following our previously defined strategy, we can now check the $F$-term potential of $\phi$ for nearby minima with $D_{\phi} W \neq 0$. Such minima may lead to higher-lying AdS vacua of (3.10) or even to metastable de Sitter. For this, we consider

$$
D_{\phi} W=K_{\phi}(\operatorname{Im} \phi) W(\phi, T)+\partial_{\phi} W_{\operatorname{sub}}(\phi) .
$$

\footnotetext{
${ }^{12}$ We disregard the dependence of $A_{K}$ on the complex structure moduli $z^{i}$ and $u / v$ since the former are stabilized at a high scale and the latter enter only in a subdominant way, i.e. as $e^{i u / v}$ with $\operatorname{Im} u / v \gg 1$. This last statement follows from analyticity and periodicity in the real direction, as is briefly mentioned in [60] and may also be established rigorously [106].
} 
If we choose the first of the two tuning options described above, where $W_{\text {sub }} \sim \epsilon$ and $\left|W_{0}+W_{\text {sub }}\right| \ll \epsilon$, a fundamental obstacle arises. Namely, in this regime generically $|W| \ll$ $\left|\partial_{\phi} W_{\text {sub }}(\phi)\right|$, such that the first term on the r.h. side of (3.12) is negligible. But the second term is holomorphic in $\phi$. Hence, by the minimum modulus principle, its absolute value cannot have a non-zero local minimum. To overcome this obstacle, a further tuning is required: we need to ensure $\left|\partial_{\phi} W_{\text {sub }}(\phi)\right| \ll\left|W_{\text {sub }}(\phi)\right|$ at the relevant point in $\phi$-space. Then one may hope that an interplay of the non-holomorphic first and the holomorphic second term on the r.h. side of (3.12) produces the desired local minimum.

If we choose the second of the two tuning options described earlier, $\left|W_{\text {sub }}\right| \ll\left|W_{0}\right| \ll \epsilon$, then it would naively appear that $(2.33)$ can produce a non-trivial local minimum of the $F$-term potential along the lines of section 2. However, things are not that simple: the extraordinary smallness of $\left|W_{\text {sub }}\right|$ comes from a compensation between different terms (e.g. the two exponentials in (2.12)), and this cancellation does in general not extend to $\partial_{\phi} W_{\text {sub. }}$ A further tuning for small $\partial_{\phi} W_{\text {sub }}$ is required. Still, the present tuning option may be advantageous since the first term on the r.h. side of (3.12) simplifies: $K_{\phi}(\operatorname{Im} \phi) W(\phi, T) \simeq K_{\phi}(\operatorname{Im} \phi)\left(W_{0}+W_{\mathrm{np}}(T)\right)$. This might allow us, similar to the scenario of section 2 , to stabilize $\operatorname{Im} \phi$ independently of $\operatorname{Re} \phi$.

Our preliminary investigation suggests that, employing either of the tuning options as discussed in the last two paragraphs, it is not straightforward to uplift KKLT. We expect that a sufficient amount of tuning freedom becomes available only if one has three or more exponential terms at one's disposal, cf. (2.33). Moreover, even after successfully engineering an $F$-term with a $(\operatorname{Re} \phi)$-dependence as in figure 1 , one is not yet finished. Namely, since the whole superfield $\phi$ is very light one must check the non-trivial additional requirement that the full potential $e^{K}\left(K^{\phi \bar{\phi}}\left|D_{\phi} W\right|^{2}-3|W|^{2}\right)$ has a local $\phi$-minimum. Analyzing these problems is beyond the scope of this work. It would lead us too far away from our basic goal of highlighting the immediate applications of the winding potential (2.24) to the challenge of uplifting.

\subsection{DGKT-type vacua}

While we derived the winding-uplift potential with a type-IIB compactification in mind, it is also possible and interesting to implement it in a type-IIA Calabi-Yau orientifold with fluxes. Specifically, we will consider DGKT vacua [57], ${ }^{13}$ following the notation of $[70,112]$. In type IIA, the Kähler moduli $T^{i}$ as well as the axio-dilaton $S=s+i \sigma$ and the complex structure moduli $U_{\lambda}=u_{\lambda}+i \nu_{\lambda}$ appear in the perturbative flux superpotential:

$$
W_{\text {flux }}=W_{\mathrm{K}}\left(T^{i}\right)+W_{\mathrm{cs}}\left(S, U_{\lambda}\right) .
$$

Here $W_{\text {cs }}$ combines the dilaton and complex-structure contributions, both involving 3 -cycle data. It takes the explicit form

$$
W_{\mathrm{cs}}\left(S, U_{\lambda}\right)=-i h_{0} S-i q^{\lambda} U_{\lambda},
$$

\footnotetext{
${ }^{13}$ Recent work includes generalizations [107, 108] as well as checks of consistency [109-111], as triggered in particular by the AdS distance (or scale separation) conjecture [58].
} 
where $h_{0}$ and $q^{\lambda}$ are independent $H_{3}$-flux numbers. Concerning $W_{K}$, it is sufficient to note that it involves terms up to cubic order in the 2-cycle variables $T^{i}$ (not to be confused with the 4-cycle Kähler moduli of type IIB, which we denoted by $T$ or $\left.T_{b}, T_{s}\right)$. The fluxes in $W_{K}$ come from Ramond-Ramond 0-, 2-, 4- and 6-form field strengths.

The Kähler potential is given by

$$
K=-\ln (8 \mathcal{V})-\ln (S+\bar{S})-2 \ln \left(\mathcal{V}^{\prime}\right)
$$

where $\mathcal{V}\left(\operatorname{Im} T^{i}\right)$ is the type-IIA CY volume. The quantity $\mathcal{V}^{\prime}$, which one may call the dual volume, is a function of the complex structure moduli $U_{\lambda}$. At large complex structure, it is defined implicitly by

$$
\mathcal{V}^{\prime} \equiv \frac{d_{\lambda \rho \sigma}}{6} v^{\lambda} v^{\rho} v^{\sigma} \quad \text { with } \quad u_{\lambda}=\partial_{v^{\lambda}} \mathcal{V}^{\prime}
$$

One may think of the $v^{\lambda}$ as 2-cycle volumes of the mirror dual type-IIB compactification [113], with $u_{\lambda}=\operatorname{Im} U_{\lambda}$ characterizing the corresponding type-IIB 4-cycles. The $d_{\lambda \rho \sigma}$ are triple intersection numbers of the mirror Calabi-Yau. We see that $\mathcal{V}^{\prime}$ is a homogeneous function of degree $\frac{3}{2}$ in $u^{\lambda}$.

The vanishing- $F$-term conditions for $S$ and $U_{\lambda}$ read

$$
\begin{aligned}
2 h_{0} s & =-\operatorname{Im} W, \\
K_{u_{\lambda}} & =-\frac{q^{\lambda}}{h_{0} s}, \\
h_{0} \sigma+q^{\lambda} \nu_{\lambda} & =-\operatorname{Re} W_{\mathrm{K}} .
\end{aligned}
$$

We do not display the corresponding SUSY equations for the Kähler moduli. Suffice it to say that the volume $\mathcal{V}$ may be considered a free parameter as it depends on the unconstrained 4-form fluxes appearing in $W_{K}$. In particular, one may go to large volume, where the following scaling behavior is found:

$$
\begin{aligned}
\left|W_{0}\right| & \sim \operatorname{Im} W_{\mathrm{K}} \sim \mathcal{V}, \quad e^{K} \sim \mathcal{V}^{-5} \\
\Rightarrow \quad V_{\mathrm{AdS}} & \sim-e^{K}\left|W_{0}\right|^{2} \sim-\mathcal{V}^{-3} .
\end{aligned}
$$

We conclude two important facts about flux-stabilized type-IIA solutions and specifically (3.17)-(3.19): first, all the real parts $u_{\lambda}$ of complex structure moduli and the dilaton field $s$ are stabilized by fluxes. In particular, the ratios $\partial_{u_{\lambda}} \mathcal{V}^{\prime} / \partial_{u_{\rho}} \mathcal{V}^{\prime}$ are determined by $H_{3}$-flux ratios $q^{\lambda} / q^{\rho}$. The overall scale is set by $s$, which is in turn fixed by (3.17). Taking into account the in general rather complicated functional relation between the variables $\partial_{u_{\lambda}} \mathcal{V}^{\prime}$ and $u_{\lambda}$, one may expect a flux discretuum like in type IIB [59]. By this we mean that the lattice of allowed flux choices translates in a sufficiently dense and random set of points on the field space parameterized by $s$ and the $u_{\lambda}$. Note that the 3 -form fluxes $h_{0}$ and $q^{\lambda}$ are subject to the $h_{+}^{2,1}+1$ tadpole cancellation conditions $m_{0} h_{0}+Q_{\mathrm{D} 6}^{0}=0$, $m_{0} q^{\lambda}+Q_{\mathrm{D} 6}^{\lambda}=0$, where $m_{0}$ is the Romans mass [57, 70]. Thus, it needs to be checked whether in a given model the flux discretuum is dense enough for our purposes. 
Second, according to (3.19) only a single linear combination of imaginary parts (the axions $\sigma$ and $\nu_{\lambda}$ ) is fixed. This ensures that a set of axions stays light, such that the winding proposal of [62] is automatically part of the DGKT setting [70].

Following [70], we now include non-perturbative corrections to (3.14):

$$
W_{\mathrm{cs}} \quad \rightarrow \quad W_{\mathrm{cs}}=W_{0}+\sum_{I} A_{I} \exp \left(-a_{0}^{I} S-\sum_{\lambda} a_{\lambda}^{I} U_{\lambda}\right)
$$

Here $I$ runs over all E2-instantons and the coefficients $a_{0}^{I} / a_{\lambda}^{I}$ specify the cycles wrapped by instanton $I$. Assuming that we are at large $s$ and $u_{\lambda}$ and that the dominant instantons contribute, this simplifies to

$$
W=W_{0}+A_{0} e^{-s} e^{-i \sigma}+\sum_{\lambda} A_{\lambda} e^{-u_{\lambda}} e^{-i \nu_{\lambda}}+\ldots
$$

We see that, including also the constraint of (3.19), this takes exactly the form of the superpotential (2.30) from our previous type-IIB analysis. The following identifications make this explicit:

$$
\begin{aligned}
u_{\lambda} & \longleftrightarrow \operatorname{Im} u_{0}^{i} \quad \text { for } \quad i=\lambda=1, \ldots, m \\
s & \longleftrightarrow \operatorname{Im} u_{0}^{0} \\
\nu_{\lambda} & \longleftrightarrow \varphi^{i} \quad \text { for } i=\lambda=1, \ldots, m \\
\sigma=-\operatorname{Re} W_{\mathrm{K}}-\sum_{\lambda=1}^{m} \frac{q^{\lambda}}{h_{0}} \nu_{\lambda} & \longleftrightarrow \operatorname{Re} u^{0}=\frac{\operatorname{Re} \psi_{0}}{N_{0}}-\sum_{i=1}^{m} \frac{N_{i}}{N_{0}} \varphi^{i} .
\end{aligned}
$$

Thus, we may now think in terms of the multi-axion potential as displayed in the last line of (2.30) and discussed at length in section 2. A key point for our uplifting application was the smallness of $\epsilon$ and the tunability of the coefficients $\mathcal{A}_{0}$ and $\mathcal{A}_{i}$. Both is ensured if, as discussed above, an appropriate discretuum in the field space of $s$ and $u_{\lambda}$ exists.

A difference to our type-IIB analysis is that here we automatically have many light axions. In section 2, a special flux choice was needed to keep one complex-structure axion light. Having several of them required more assumptions. We expect that there is nothing wrong with realizing our winding uplift in the type-IIA case in a multi-axion situation. But, as discussed in [70], it is also easy to return to the single-axion case analyzed in detail before.

First, we can choose a CY with a small number of complex structure moduli. This may however not be the optimal path since it is expected that in such models the flux discretuum is also smaller.

Second, we can choose fluxes implementing a hierarchy in the saxion values. If, for example, $s, u_{1} \gg u_{\lambda}$ for $\lambda>1$, we find $\mathcal{A}_{i} \gg 1$ for $i=2, \ldots, m$. As a result, all $\nu_{\lambda}$ for $\lambda>1$ are stabilized (supersymmetrically) at a higher scale. Only the lightest axion(s), in our case a linear combination of $\sigma$ and $\nu_{1}$, will remain relevant, experiencing an effective winding potential which follows from (2.30). It can be recast in the simpler form (2.24), which we have studied in great detail.

As a result, we expect that non-zero minima of the complex-structure $F$-term potential arise for appropriate choices of $\mathrm{CY}$ and flux. As discussed in the beginning of this section, 
we should be able to parametrically separate the potential difference between false and true vacuum, $\Delta V \sim e^{K} \epsilon^{2} \gamma^{2}$, and the potential barrier $\sim e^{K} \epsilon^{2}$. In our regime of parametric control, $\epsilon \ll\left|W_{0}\right|$, the uplift remains small compared to the depth $e^{K}\left|W_{0}\right|^{2}$ of the DGKT AdS vacuum. So, consistently with the no-go theorem of $[114,115]$, dS is out of reach.

However, due to the parametric separation between uplift and potential barrier, the brane tension $T$ between true and false vacuum may be too large for a bubble of true vacuum to nucleate, $T / M_{\mathrm{P}}>\sqrt{4 / 3}\left(\sqrt{\left|V_{t}\right|}-\sqrt{\left|V_{f}\right|}\right)[47,48,116]$. The resulting non-SUSY AdS vacuum would be absolutely stable against the Coleman-de Luccia decay [92] to the underlying SUSY AdS vacuum. If no other decay path exists, and we see no obvious candidate in the present setting, this would provide a counterexample to the non-SUSY AdS conjecture. Crucially, given the minimalist set of ingredients in our construction, it may actually be possible to study the type-IIA flux landscape in concrete models and establish, using e.g. the technology developed in [60], that the small uplifts we propose really exist.

\section{Conclusion}

We have presented a mechanism for metastable SUSY breaking in the landscape. Based on the winding scenario of [62], we have described how the interplay of multiple periodic terms in the complex-structure super- and Kähler potential can lead to an $F$-term scalar potential with non-trivial local minima. Crucially, we have argued that the tuning power of the complex-structure landscape can be used to ensure that these minima are at parametrically small value of the potential, resulting in long-lived vacua. As applications, we discussed the uplift of LVS, DGKT and KKLT AdS vacua.

KKLT is the most difficult case. Here, the requirement of a small perturbative superpotential $W_{0}$ inhibits the straightforward application of our simplest setup from section 2 . The problem is that not only an axionic component $\operatorname{Re}(\phi)$, but the full complex field $\phi$ remains light. While we described how our mechanism may still work, using a specific tuning of $W_{0}$ and of the additional periodic terms, we had to leave the explicit calculation of the minimum value and stability of the $\operatorname{Im}(\phi)$-component for future work.

For supersymmetric DGKT AdS vacua, our SUSY breaking mechanism appears to be very robust. Compared to KKLT, the construction requires less tuning. In fact, the DGKT setting naturally gives rise to a multi-axion potential which also comes with more tunable parameters. As a remark of caution, we note that the tuning power of the type-IIA complex structure landscape is less established than its type-IIB counterpart. (A brief discussion of how the necessary tuning could be implemented appears below (3.20).) Assuming that this concern can be dispelled, we may have found stable non-supersymmetric AdS solutions serving as counterexamples to the non-SUSY AdS conjecture [44].

Finally, we showed that the application of our winding uplift in the LVS context is straightforward. Of course, in this case one is working on the basis of a non-supersymmetric AdS solution, which may in itself already be in conflict with the non-SUSY AdS conjecture (cf. the discussion in [102]). If the LVS AdS vacuum exists, our uplift provides a new route to metastable dS vacua, challenging the de Sitter conjecture [29]. The strong point of this 
uplift is its conceptual and technical simplicity, allowing in principle for the computerized search for a completely explicit example. A summary of the main tuning requirements that have to be met appears at the end of section 2.3. We want to highlight the very recent work of [91] where the parameters $\alpha$ and $\epsilon$ of (2.24) were made explicit in a CICY setup. Unfortunately this CICY construction does not allow for an implementation of the LVS, so some more work needs to be done.

Before closing, we recall that we started our work with a discussion of possible extended swampland conjectures against non-SUSY AdS. We do not want to repeat this discussion but only emphasize one possible landscape-skeptical scenario: if the LVS AdS vacuum and the KKLT uplift (by our or other methods) fail, then our only application is DGKT. Here, our uplift to non-SUSY AdS may work, but it appears difficult to raise the $F$-term scale far above the AdS scale. Such a situation would support a 'Strong-SUSY-Breaking Conjecture', excluding non-SUSY AdS vacua (stable or metastable) with an $F$-term scale parametrically above the AdS scale. This conjecture is interesting as it rules out a type of cosmology which would otherwise be perfectly consistent with most of the swampland conjectures and with observers like us: namely, a universe just like ours but where the late stages of cosmology are governed by a tiny negative cosmological constant. If one takes the landscape/multiverse view on fundamental physics seriously (see [117] for a recent review), the existence or non-existence of such cosmologies in string theory represents an important question.

\section{Acknowledgments}

We would like to thank Daniel Junghans for useful discussions. A.H. acknowledges an enjoyable conversation with Alexander Westphal about possible swampland conjectures against SUSY breaking which has inspired some of the ideas discussed in this paper. This work is supported by the Deutsche Forschungsgemeinschaft (DFG, German Research Foundation) under Germany's Excellence Strategy EXC 2181/1 - 390900948 (the Heidelberg STRUCTURES Excellence Cluster) and the Graduiertenkolleg 'Particle physics beyond the Standard Model' (GRK 1940).

Open Access. This article is distributed under the terms of the Creative Commons Attribution License (CC-BY 4.0), which permits any use, distribution and reproduction in any medium, provided the original author(s) and source are credited.

\section{References}

[1] S. Kachru, R. Kallosh, A.D. Linde and S.P. Trivedi, de Sitter vacua in string theory, Phys. Rev. D 68 (2003) 046005 [hep-th/0301240] [InSPIRE].

[2] V. Balasubramanian, P. Berglund, J.P. Conlon and F. Quevedo, Systematics of moduli stabilisation in Calabi-Yau flux compactifications, JHEP 03 (2005) 007 [hep-th/0502058] [INSPIRE].

[3] A. Saltman and E. Silverstein, The Scaling of the no scale potential and de Sitter model building, JHEP 11 (2004) 066 [hep-th/0402135] [INSPIRE]. 
[4] M. Gomez-Reino and C.A. Scrucca, Locally stable non-supersymmetric Minkowski vacua in supergravity, JHEP 05 (2006) 015 [hep-th/0602246] [INSPIRE].

[5] O. Lebedev, H.P. Nilles and M. Ratz, de Sitter vacua from matter superpotentials, Phys. Lett. B 636 (2006) 126 [hep-th/0603047] [INSPIRE].

[6] M. Dine, R. Kitano, A. Morisse and Y. Shirman, Moduli decays and gravitinos, Phys. Rev. D 73 (2006) 123518 [hep-ph/0604140] [INSPIRE].

[7] F. Brummer, A. Hebecker and M. Trapletti, SUSY breaking mediation by throat fields, Nucl. Phys. B $\mathbf{7 5 5}$ (2006) 186 [hep-th/0605232] [INSPIRE].

[8] E. Dudas, C. Papineau and S. Pokorski, Moduli stabilization and uplifting with dynamically generated F-terms, JHEP 02 (2007) 028 [hep-th/0610297] [INSPIRE].

[9] R. Kallosh and A.D. Linde, O'KKLT, JHEP 02 (2007) 002 [hep-th/0611183] [InSPIRE].

[10] H. Abe, T. Higaki, T. Kobayashi and Y. Omura, Moduli stabilization, F-term uplifting and soft supersymmetry breaking terms, Phys. Rev. D 75 (2007) 025019 [hep-th/0611024] [INSPIRE].

[11] H. Abe, T. Higaki and T. Kobayashi, More about F-term uplifting, Phys. Rev. D 76 (2007) 105003 [arXiv: 0707.2671] [INSPIRE].

[12] A. Achucarro and K. Sousa, F-term uplifting and moduli stabilization consistent with Kähler invariance, JHEP 03 (2008) 002 [arXiv:0712.3460] [INSPIRE].

[13] M. Cicoli, D. Klevers, S. Krippendorf, C. Mayrhofer, F. Quevedo and R. Valandro, Explicit de Sitter Flux Vacua for Global String Models with Chiral Matter, JHEP 05 (2014) 001 [arXiv: 1312.0014] [INSPIRE].

[14] M. Cicoli, F. Quevedo and R. Valandro, de Sitter from T-branes, JHEP 03 (2016) 141 [arXiv: 1512.04558] [INSPIRE].

[15] E.A. Bergshoeff, K. Dasgupta, R. Kallosh, A. Van Proeyen and T. Wrase, $\overline{\mathrm{D} 3}$ and $d S$, JHEP 05 (2015) 058 [arXiv: 1502.07627] [INSPIRE].

[16] A. Retolaza and A. Uranga, de Sitter Uplift with Dynamical SUSY Breaking, JHEP 04 (2016) 137 [arXiv:1512.06363] [INSPIRE].

[17] R. Argurio et al., The Octagon and the Non-Supersymmetric String Landscape, Phys. Lett. $B \mathbf{8 1 5}$ (2021) 136153 [arXiv: 2005.09671] [InSPIRE].

[18] R. Argurio et al., Dimers, Orientifolds and Stability of Supersymmetry Breaking Vacua, JHEP 01 (2021) 061 [arXiv:2007.13762] [INSPIRE].

[19] C.P. Burgess, R. Kallosh and F. Quevedo, de Sitter string vacua from supersymmetric D terms, JHEP 10 (2003) 056 [hep-th/0309187] [INSPIRE].

[20] G. Villadoro and F. Zwirner, De-Sitter vacua via consistent D-terms, Phys. Rev. Lett. 95 (2005) 231602 [hep-th/0508167] [INSPIRE].

[21] A. Achucarro, B. de Carlos, J.A. Casas and L. Doplicher, de Sitter vacua from uplifting D-terms in effective supergravities from realistic strings, JHEP 06 (2006) 014 [hep-th/0601190] [INSPIRE].

[22] E. Dudas and Y. Mambrini, Moduli stabilization with positive vacuum energy, JHEP 10 (2006) 044 [hep-th/0607077] [INSPIRE].

[23] M. Haack, D. Krefl, D. Lüst, A. Van Proeyen and M. Zagermann, Gaugino Condensates and D-terms from D7-branes, JHEP 01 (2007) 078 [hep-th/0609211] [INSPIRE]. 
[24] D. Cremades, M.P. Garcia del Moral, F. Quevedo and K. Suruliz, Moduli stabilisation and de Sitter string vacua from magnetised D7 branes, JHEP 05 (2007) 100 [hep-th/0701154] [INSPIRE].

[25] S. Krippendorf and F. Quevedo, Metastable SUSY Breaking, de Sitter Moduli Stabilisation and Kähler Moduli Inflation, JHEP 11 (2009) 039 [arXiv:0901.0683] [INSPIRE].

[26] U.H. Danielsson and T. Van Riet, What if string theory has no de Sitter vacua?, Int. J. Mod. Phys. D 27 (2018) 1830007 [arXiv:1804.01120] [INSPIRE].

[27] G. Obied, H. Ooguri, L. Spodyneiko and C. Vafa, de Sitter Space and the Swampland, arXiv: 1806.08362 [INSPIRE].

[28] S.K. Garg and C. Krishnan, Bounds on Slow Roll and the de Sitter Swampland, JHEP 11 (2019) 075 [arXiv: 1807.05193] [INSPIRE].

[29] H. Ooguri, E. Palti, G. Shiu and C. Vafa, Distance and de Sitter Conjectures on the Swampland, Phys. Lett. B $\mathbf{7 8 8}$ (2019) 180 [arXiv:1810.05506] [INSPIRE].

[30] F. Carta, J. Moritz and A. Westphal, Gaugino condensation and small uplifts in KKLT, JHEP 08 (2019) 141 [arXiv:1902.01412] [INSPIRE].

[31] X. Gao, A. Hebecker and D. Junghans, Control issues of KKLT, arXiv:2009.03914 [INSPIRE].

[32] J. Moritz, A. Retolaza and A. Westphal, Toward de Sitter space from ten dimensions, Phys. Rev. D 97 (2018) 046010 [arXiv: 1707.08678] [INSPIRE].

[33] F.F. Gautason, V. Van Hemelryck and T. Van Riet, The Tension between 10D Supergravity and dS Uplifts, Fortsch. Phys. 67 (2019) 1800091 [arXiv:1810.08518] [InSPIRE].

[34] Y. Hamada, A. Hebecker, G. Shiu and P. Soler, On brane gaugino condensates in 10d, JHEP 04 (2019) 008 [arXiv: 1812.06097] [INSPIRE].

[35] R. Kallosh, A. Linde, E. McDonough and M. Scalisi, dS Vacua and the Swampland, JHEP 03 (2019) 134 [arXiv: 1901.02022] [inSPIRE].

[36] Y. Hamada, A. Hebecker, G. Shiu and P. Soler, Understanding KKLT from a 10d perspective, JHEP 06 (2019) 019 [arXiv: 1902.01410] [INSPIRE].

[37] F.F. Gautason, V. Van Hemelryck, T. Van Riet and G. Venken, A 10d view on the KKLT AdS vacuum and uplifting, JHEP 06 (2020) 074 [arXiv: 1902.01415] [INSPIRE].

[38] I. Bena, E. Dudas, M. Graña and S. Lüst, Uplifting Runaways, Fortsch. Phys. 67 (2019) 1800100 [arXiv: 1809.06861] [INSPIRE].

[39] R. Blumenhagen, D. Kläwer and L. Schlechter, Swampland Variations on a Theme by KKLT, JHEP 05 (2019) 152 [arXiv: 1902.07724] [INSPIRE].

[40] L. Randall, The Boundaries of KKLT, Fortsch. Phys. 68 (2020) 1900105 [arXiv: 1912.06693] [INSPIRE].

[41] I. Bena, J. Blåbäck, M. Graña and S. Lüst, The Tadpole Problem, arXiv:2010.10519 [INSPIRE].

[42] C. Crinò, F. Quevedo and R. Valandro, On de Sitter String Vacua from Anti-D3-branes in the Large Volume Scenario, arXiv:2010.15903 [INSPIRE].

[43] S. Sethi, Supersymmetry Breaking by Fluxes, JHEP 10 (2018) 022 [arXiv:1709.03554] [INSPIRE]. 
[44] H. Ooguri and C. Vafa, Non-supersymmetric AdS and the Swampland, Adv. Theor. Math. Phys. 21 (2017) 1787 [arXiv:1610.01533] [INSPIRE].

[45] B. Freivogel and M. Kleban, Vacua Morghulis, arXiv:1610.04564 [INSPIRE].

[46] G.T. Horowitz, J. Orgera and J. Polchinski, Nonperturbative Instability of $A d S_{5} \times S^{5} / Z_{k}$, Phys. Rev. D 77 (2008) 024004 [arXiv:0709.4262] [INSPIRE].

[47] D. Harlow, Metastability in Anti de Sitter Space, arXiv:1003.5909 [INSPIRE].

[48] P. Narayan and S.P. Trivedi, On The Stability Of Non-Supersymmetric AdS Vacua, JHEP 07 (2010) 089 [arXiv: 1002.4498] [INSPIRE].

[49] A. Almuhairi and J. Polchinski, Magnetic AdS $\times \mathbb{R}^{2}$ : Supersymmetry and stability, arXiv:1108.1213 [INSPIRE].

[50] A. Guarino, J. Tarrio and O. Varela, Brane-jet stability of non-supersymmetric AdS vacua, JHEP 09 (2020) 110 [arXiv: 2005.07072] [INSPIRE].

[51] A. Guarino, E. Malek and H. Samtleben, Stable Nonsupersymmetric Anti-de Sitter Vacua of Massive IIA Supergravity, Phys. Rev. Lett. 126 (2021) 061601 [arXiv:2011.06600] [INSPIRE].

[52] T. Fischbacher, K. Pilch and N.P. Warner, New Supersymmetric and Stable, Non-Supersymmetric Phases in Supergravity and Holographic Field Theory, arXiv: 1010.4910 [INSPIRE].

[53] E. Silverstein, (A)dS backgrounds from asymmetric orientifolds, Clay Mat. Proc. 1 (2002) 179 [hep-th/0106209] [INSPIRE].

[54] A. Maloney, E. Silverstein and A. Strominger, de Sitter space in noncritical string theory, in Workshop on Conference on the Future of Theoretical Physics and Cosmology in Honor of Steven Hawking's 60th Birthday, (2002) [hep-th/0205316] [INSPIRE].

[55] S. Abel, K.R. Dienes and E. Mavroudi, Towards a nonsupersymmetric string phenomenology, Phys. Rev. D 91 (2015) 126014 [arXiv:1502.03087] [INSPIRE].

[56] E. Hardy and S. Parameswaran, Thermal Dark Energy, Phys. Rev. D 101 (2020) 023503 [arXiv: 1907.10141] [INSPIRE].

[57] O. DeWolfe, A. Giryavets, S. Kachru and W. Taylor, Type IIA moduli stabilization, JHEP 07 (2005) 066 [hep-th/0505160] [INSPIRE].

[58] D. Lüst, E. Palti and C. Vafa, AdS and the Swampland, Phys. Lett. B 797 (2019) 134867 [arXiv:1906.05225] [INSPIRE].

[59] F. Denef and M.R. Douglas, Distributions of flux vacua, JHEP 05 (2004) 072 [hep-th/0404116] [INSPIRE].

[60] M. Demirtas, M. Kim, L. Mcallister and J. Moritz, Vacua with Small Flux Superpotential, Phys. Rev. Lett. 124 (2020) 211603 [arXiv: 1912.10047] [INSPIRE].

[61] J.E. Kim, H.P. Nilles and M. Peloso, Completing natural inflation, JCAP 01 (2005) 005 [hep-ph/0409138] [INSPIRE].

[62] A. Hebecker, P. Mangat, F. Rompineve and L.T. Witkowski, Winding out of the Swamp: Evading the Weak Gravity Conjecture with F-term Winding Inflation?, Phys. Lett. B 748 (2015) 455 [arXiv: 1503.07912] [INSPIRE].

[63] T. Kobayashi, A. Oikawa and H. Otsuka, New potentials for string axion inflation, Phys. Rev. D 93 (2016) 083508 [arXiv: 1510.08768] [INSPIRE]. 
[64] N. Cabo Bizet, O. Loaiza-Brito and I. Zavala, Mirror quintic vacua: hierarchies and inflation, JHEP 10 (2016) 082 [arXiv: 1605. 03974] [INSPIRE].

[65] R. Blumenhagen, D. Herschmann and F. Wolf, String Moduli Stabilization at the Conifold, JHEP 08 (2016) 110 [arXiv: 1605. 06299] [INSPIRE].

[66] R. Blumenhagen, D. Herschmann and F. Wolf, Challenges for Moduli Stabilization and String Cosmology near the Conifold, PoS CORFU2016 (2017) 104 [arXiv:1704.04140] [INSPIRE].

[67] A. Hebecker, P. Mangat, F. Rompineve and L.T. Witkowski, Tuning and Backreaction in F-term Axion Monodromy Inflation, Nucl. Phys. B 894 (2015) 456 [arXiv:1411.2032] [INSPIRE].

[68] A. Hebecker, S. Leonhardt, J. Moritz and A. Westphal, Thraxions: Ultralight Throat Axions, JHEP 04 (2019) 158 [arXiv:1812.03999] [INSPIRE].

[69] J. March-Russell and R. Petrossian-Byrne, QCD, Flavor, and the de Sitter Swampland, arXiv:2006.01144 [INSPIRE].

[70] E. Palti, On Natural Inflation and Moduli Stabilisation in String Theory, JHEP 10 (2015) 188 [arXiv: 1508.00009] [INSPIRE].

[71] A. Westphal, de Sitter string vacua from Kähler uplifting, JHEP 03 (2007) 102 [hep-th/0611332] [INSPIRE].

[72] S. de Alwis and K. Givens, Physical Vacua in IIB Compactifications with a Single Kähler Modulus, JHEP 10 (2011) 109 [arXiv:1106.0759] [INSPIRE].

[73] M. Rummel and A. Westphal, A sufficient condition for de Sitter vacua in type IIB string theory, JHEP 01 (2012) 020 [arXiv:1107.2115] [INSPIRE].

[74] M. Cicoli, A. Maharana, F. Quevedo and C.P. Burgess, de Sitter String Vacua from Dilaton-dependent Non-perturbative Effects, JHEP 06 (2012) 011 [arXiv:1203.1750] [INSPIRE].

[75] J. Louis, M. Rummel, R. Valandro and A. Westphal, Building an explicit de Sitter, JHEP 10 (2012) 163 [arXiv: 1208.3208] [INSPIRE].

[76] J. Blåbäck, D. Roest and I. Zavala, de Sitter Vacua from Nonperturbative Flux Compactifications, Phys. Rev. D 90 (2014) 024065 [arXiv:1312.5328] [InSPIRE].

[77] M. Rummel and Y. Sumitomo, de Sitter Vacua from a D-term Generated Racetrack Uplift, JHEP 01 (2015) 015 [arXiv: 1407.7580] [INSPIRE].

[78] A.P. Braun, M. Rummel, Y. Sumitomo and R. Valandro, de Sitter vacua from a D-term generated racetrack potential in hypersurface Calabi-Yau compactifications, JHEP 12 (2015) 033 [arXiv: 1509.06918] [INSPIRE].

[79] A. Hebecker, S.C. Kraus and L.T. Witkowski, D7-Brane Chaotic Inflation, Phys. Lett. B 737 (2014) 16 [arXiv:1404.3711] [InSPIRE].

[80] M. Arends et al., D7-Brane Moduli Space in Axion Monodromy and Fluxbrane Inflation, Fortsch. Phys. 62 (2014) 647 [arXiv:1405.0283] [InSPIRE].

[81] L. McAllister, E. Silverstein, A. Westphal and T. Wrase, The Powers of Monodromy, JHEP 09 (2014) 123 [arXiv: 1405.3652] [INSPIRE].

[82] R. Blumenhagen, D. Herschmann and E. Plauschinn, The Challenge of Realizing F-term Axion Monodromy Inflation in String Theory, JHEP 01 (2015) 007 [arXiv:1409.7075] [INSPIRE]. 
[83] H. Hayashi, R. Matsuda and T. Watari, Issues in Complex Structure Moduli Inflation, arXiv: 1410.7522 [INSPIRE].

[84] H. Abe, T. Kobayashi and H. Otsuka, Natural inflation with and without modulations in type IIB string theory, JHEP 04 (2015) 160 [arXiv:1411.4768] [INSPIRE].

[85] I. García-Etxebarria, T.W. Grimm and I. Valenzuela, Special Points of Inflation in Flux Compactifications, Nucl. Phys. B 899 (2015) 414 [arXiv: 1412.5537] [INSPIRE].

[86] F. Baume and E. Palti, Backreacted Axion Field Ranges in String Theory, JHEP 08 (2016) 043 [arXiv: 1602.06517] [INSPIRE].

[87] A. Hebecker, D. Junghans and A. Schachner, Large Field Ranges from Aligned and Misaligned Winding, JHEP 03 (2019) 192 [arXiv:1812.05626] [INSPIRE].

[88] S. Hosono, A. Klemm and S. Theisen, Lectures on mirror symmetry, Lect. Notes Phys. 436 (1994) 235 [hep-th/9403096] [INSPIRE].

[89] T.W. Grimm and J. Louis, The Effective action of $N=1$ Calabi-Yau orientifolds, Nucl. Phys. B 699 (2004) 387 [hep-th/0403067] [INSPIRE].

[90] P. Corvilain, T.W. Grimm and D. Regalado, Shift-symmetries and gauge coupling functions in orientifolds and F-theory, JHEP 05 (2017) 059 [arXiv:1607.03897] [INSPIRE].

[91] F. Carta, A. Mininno, N. Righi and A. Westphal, Gopakumar-Vafa Hierarchies in Winding Inflation and Uplifts, arXiv:2101.07272 [INSPIRE].

[92] S.R. Coleman and F. De Luccia, Gravitational Effects on and of Vacuum Decay, Phys. Rev. D 21 (1980) 3305 [INSPIRE].

[93] E. Witten, Instability of the Kaluza-Klein Vacuum, Nucl. Phys. B 195 (1982) 481 [inSPIRE].

[94] I. García Etxebarria, M. Montero, K. Sousa and I. Valenzuela, Nothing is certain in string compactifications, JHEP 12 (2020) 032 [arXiv: 2005.06494] [INSPIRE].

[95] D. Gallego, On the Effective Description of Large Volume Compactifications, JHEP 06 (2011) 087 [arXiv: 1103.5469] [INSPIRE].

[96] M. Cicoli, S. Krippendorf, C. Mayrhofer, F. Quevedo and R. Valandro, D-Branes at del Pezzo Singularities: Global Embedding and Moduli Stabilisation, JHEP 09 (2012) 019 [arXiv:1206.5237] [INSPIRE].

[97] D. Gallego, M.C.D. Marsh, B. Vercnocke and T. Wrase, A New Class of de Sitter Vacua in Type IIB Large Volume Compactifications, JHEP 10 (2017) 193 [arXiv:1707.01095] [INSPIRE].

[98] R. Kallosh, A. Linde, B. Vercnocke and T. Wrase, Analytic Classes of Metastable de Sitter Vacua, JHEP 10 (2014) 011 [arXiv:1406.4866] [InSPIRE].

[99] M.C.D. Marsh, B. Vercnocke and T. Wrase, Decoupling and de Sitter Vacua in Approximate No-Scale Supergravities, JHEP 05 (2015) 081 [arXiv:1411.6625] [INSPIRE].

[100] K. Becker, M. Becker, M. Haack and J. Louis, Supersymmetry breaking and $\alpha^{\prime}$-corrections to flux induced potentials, JHEP 06 (2002) 060 [hep-th/0204254] [INSPIRE].

[101] S. de Alwis, R. Gupta, E. Hatefi and F. Quevedo, Stability, Tunneling and Flux Changing de Sitter Transitions in the Large Volume String Scenario, JHEP 11 (2013) 179 [arXiv:1308.1222] [INSPIRE].

[102] J.P. Conlon and F. Quevedo, Putting the Boot into the Swampland, JHEP 03 (2019) 005 [arXiv: 1811.06276] [INSPIRE]. 
[103] A. Strominger, S.-T. Yau and E. Zaslow, Mirror symmetry is T duality, Nucl. Phys. B 479 (1996) 243 [hep-th/9606040] [INSPIRE].

[104] M. Kontsevich and Y. Soibelman, Homological mirror symmetry and torus fibrations, in KIAS Annual International Conference on Symplectic Geometry and Mirror Symmetry, (2000) [math/0011041] [INSPIRE].

[105] M. Gross and P.M.H. Wilson, Large complex structure limits of K3 surfaces, J. Differ. Geom. 55 (2000) 475.

[106] A. Hebecker, J. Moritz and A. Schachner, unpublished (2020).

[107] F. Marchesano and J. Quirant, A Landscape of AdS Flux Vacua, JHEP 12 (2019) 110 [arXiv: 1908.11386] [INSPIRE].

[108] T.W. Grimm, C. Li and I. Valenzuela, Asymptotic Flux Compactifications and the Swampland, JHEP 06 (2020) 009 [Erratum ibid. 01 (2021) 007] [arXiv:1910.09549] [INSPIRE].

[109] D. Junghans, O-Plane Backreaction and Scale Separation in Type IIA Flux Vacua, Fortsch. Phys. 68 (2020) 2000040 [arXiv: 2003.06274] [INSPIRE].

[110] F. Marchesano, E. Palti, J. Quirant and A. Tomasiello, On supersymmetric AdS $S_{4}$ orientifold vacua, JHEP 08 (2020) 087 [arXiv: 2003.13578] [INSPIRE].

[111] S. Baines and T. Van Riet, Smearing orientifolds in flux compactifications can be OK, Class. Quant. Grav. 37 (2020) 195015 [arXiv: 2005.09501] [INSPIRE].

[112] E. Palti, G. Tasinato and J. Ward, WEAKLY-coupled IIA Flux Compactifications, JHEP 06 (2008) 084 [arXiv:0804.1248] [INSPIRE].

[113] T.W. Grimm and J. Louis, The Effective action of type IIA Calabi-Yau orientifolds, Nucl. Phys. B 718 (2005) 153 [hep-th/0412277] [INSPIRE].

[114] M.P. Hertzberg, S. Kachru, W. Taylor and M. Tegmark, Inflationary Constraints on Type IIA String Theory, JHEP 12 (2007) 095 [arXiv: 0711.2512] [InSPIRE].

[115] R. Flauger, S. Paban, D. Robbins and T. Wrase, Searching for slow-roll moduli inflation in massive type IIA supergravity with metric fluxes, Phys. Rev. D 79 (2009) 086011 [arXiv:0812.3886] [INSPIRE].

[116] M. Cvetič, S. Griffies and S.-J. Rey, Nonperturbative stability of supergravity and superstring vacua, Nucl. Phys. B 389 (1993) 3 [hep-th/9206004] [INSPIRE].

[117] A. Hebecker, Lectures on Naturalness, String Landscape and Multiverse, arXiv:2008.10625 [INSPIRE]. 